\title{
EFFECT OF THE COVID-19 PANDEMIC IN A MASTOLOGY SERVICE AT A TERTIARY HOSPITAL
}

Alexandre Villela de Freitas'1, Arnaldo Pereira Cortez Junior ${ }^{1}$, Marília Silva de Souza Takimoto1, Vanessa Radavelli de Araujo', Carolina Rosa de Araujo ${ }^{1}$

${ }^{1}$ Hospital Ministro Costa Cavalcanti - Foz do Iguaçu (PR), Brazil.

Introduction: The measures of social isolation and campaigns to combat the COVID-19 pandemic released after March 2020 and the risk of severe respiratory disease in the population older than 65 years determined a decrease in the performance of preventive tests and demand to outpatient clinics, as well as the removal of professionals in risk groups reducing the supply of care. The delay in diagnosis and initiation of treatment is considered a problem in health systems worldwide, directly impacting mortality from the disease. In force since 2012, Lawn. 12732 of the Brazilian Department of Health establishes that the first cancer treatment for SUS (Brazilian Unified Health System) patients must begin within a maximum of 60 days from the signature of the pathological report that confirms malignant neoplasia. Objectives: To evaluate the effects of the pandemic in a mastology service at a tertiary hospital of the Unified Health System (SUS) from the measurement of the interval between diagnosis and initiation of treatment, first proposed oncological, surgical (CT) or systemic (TS) treatment and the justifications pointed out when the beginning of treatment occurred in a period of more than sixty days. Methods: Cross-sectional cohort study of patients diagnosed with breast cancer between March 2019 and January 2021, divided into two groups - prepandemic and pandemic from the evaluation of data in the hospital management system (Stratec) and comparison of information between the two groups. Results: In group 1, prepandemic, from March 2019 to March 2020, 82 patients admitted, mean of 6.3 patients per month, 62 started treatment before 60 days (59TC and 3TS) and 20 after (13TC and 7TS). In group 2, pandemic, from April 2020 to January 2021, 65 patients, mean of 7.2 patients per month, 51 started treatment before 60 days (37TC and 14TS) and 14 after (11TC and 3TS), 32.3 and 27.5\%, respectively. Regarding the indication of initial treatment, surgical treatment predominated in both groups, however there was an increase in the indication of systemic treatment in group 2 (17/65 vs 10/82). Among the main justifications for delay in the beginning of treatment, we identified clinical conditions of the patients, the need for a plastic surgery team and unavailability of a vacancy in the operating room in the first group, and clinical conditions of the patients and indecision to accept the therapeutic proposal in the second group. Conclusions: The reception by the nursing team to the patients at the time of diagnosis guiding the performance of preoperative examinations and consultations, availability of the operating room exclusively for emergencies, cardiac or oncological surgeries and replacement of professionals when the absence was necessary were effective measures in the maintenance of care and quality of service. 